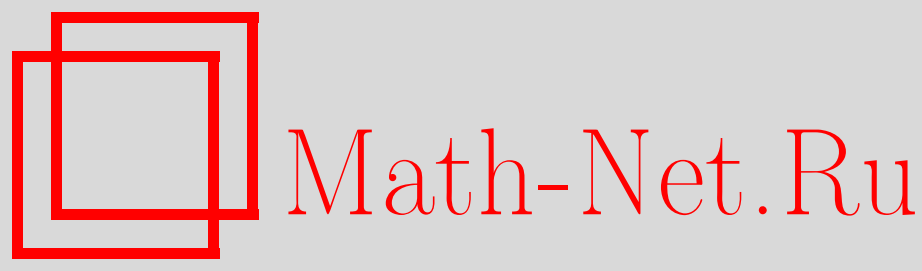

А. Кудлис, А. И. Соколов, Теория поля и анизотропия кубического ферромагнетика вблизи точки Кюри, ТМФ, 2017, том 190, номер 2, 344-353

DOI: https://doi.org/10.4213/tmf9112

Использование Общероссийского математического портала Math-Net.Ru подразумевает, что вы прочитали и согласны с пользовательским соглашением http://www . mathnet.ru/rus/agreement

Параметры загрузки:

IP : 54.224 .187 .69

26 апреля 2023 г., 12:38:37

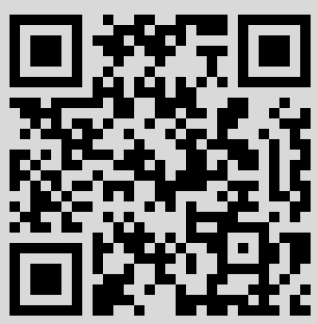




\title{
ТЕОРИЯ ПОЛЯ И АНИЗОТРОПИЯ КУБИЧЕСКОГО ФЕРРОМАГНЕТИКА ВБЛИЗИ ТОЧКИ КЮРИ
}

\begin{abstract}
Известно, что критические флуктуации могут изменять эффективную анизотропию кубического ферромагнетика вблизи точки Кюри. Если кристалл испытывает фазовый переход в орторомбическую фазу и его исходная анизотропия не слишком велика, эффективная анизотропия принимает в точке $T_{\mathrm{c}}$ универсальное значение $A^{*}=v^{*} / u^{*}$, где $u^{*}$ и $v^{*}$ - координаты кубической фиксированной точки уравнений ренормгруппы, входящие в скейлинговое уравнение состояния и в выражения для нелинейных восприимчивостей. С помощью метода псевдо- $\epsilon$-разложения найдено численное значение параметра анизотропии $A$ в критической точке. Суммирование по Паде шестипетлевых псевдо- $\epsilon-$ разложений для $u^{*}, v^{*}$ и $A^{*}$ приводит к оценке $A^{*}=0.13 \pm 0.01$, свидетельствующей о том, что обнаружение анизотропного критического поведения кубических ферромагнетиков в физических и компьютерных экспериментах является вполне возможным.
\end{abstract}

Ключевые слова: кубическая модель, эффективная анизотропия, ренормализационная группа, $\epsilon$-разложение, псевдо- $\epsilon$-разложение.

DOI: $10.4213 / \operatorname{tmf} 9112$

При подходе к критической точке тепловые флуктуации намагниченности становятся настолько сильными, что эффективная анизотропия кубического ферромагнетика начинает ощутимо зависеть от температуры. Конкретно, речь идет о кристаллической анизотропии нелинейных восприимчивостей различных порядков. Если в кристалле происходит фазовый переход в орторомбическую фазу и исходная ани-

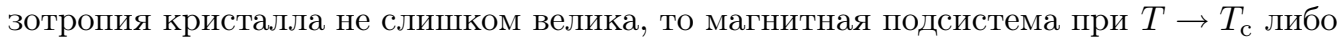

Работа А.Я. Кудлиса была выполнена в рамках проекта № 16-11-10218 Российского научного фонда. А.И. Соколов благодарит за поддержку РФФИ (грант № 15-02-04687).

* Санкт-Петербургский государственный университет, Санкт-Петербург, Старый Петергоф, Россия. E-mail: ais2002@mail.ru

${ }^{\dagger}$ Санкт-Петербургский национальный исследовательский университет информационных технологий, механики и оптики, Санкт-Петербург, Россия 
становится изотропной [1], либо ее анизотропия $A$ выходит на некоторое универсальное значение, не зависящее от величины $A$ вдали от точки Кюри. При больших затравочных значениях $A$ флуктуации делают критическое поведение системы неустойчивым, и в ней происходит фазовый переход первого рода [2]-[4]. Какой из описанных выше сценариев реализуется в критической области, зависит от числа компонент (размерности) параметра порядка $n$. Если $n<n_{\mathrm{c}}$, то система выходит на изотропную критическую асимптотику, если же $n>n_{\mathrm{c}}$, она остается анизотропной в точке Кюри [5].

Ясно, что численное значение граничной размерности $n_{\mathrm{c}}$ представляет первостепенный физический интерес, поскольку именно оно определяет, как будут вести себя реальные кубические $(n=3)$ и тетрагональные $(n=2)$ ферромагнетики при температурах вблизи $T_{c}$. Ренормгрупповой (РГ) анализ в низших порядках теории возмущений [3], [6], [7], а также некоторые расчеты на решетках [8] и применение техники непертурбативной РГ [9] показали, что $n_{\mathrm{c}}$, скорее всего, лежит между 3 и 4. Однако дальнейшие исследования, в том числе пересуммирование трехпетлевых РГ-разложений [10], [11] и многопетлевые РГ-расчеты для трехмерной [12]-[14] и $(4-\epsilon)$-мерной [15] моделей, изменили эту оценку на $n_{\mathrm{c}}<3$ [10], [11]-[22]. Так, детальный трехмерный РГ-анализ [14], обработка различными способами пятипетлевых $\epsilon$-разложений [18], [14] и обращение к технике псевдо- $\epsilon$-разложения [20] дали соответственно $n_{\mathrm{c}}=2.89, n_{\mathrm{c}}=2.855, n_{\mathrm{c}}=2.87$ и $n_{\mathrm{c}}=2.862$. Это означает, что кубические ферромагнетики, испытывающие фазовые переходы второго рода, должны принадлежать к особому - кубическому - классу универсальности, т. е. обладать конечной анизотропией в точке Кюри и иметь свой специфический набор критических индексов.

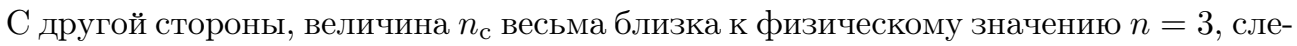
довательно, кубическая фиксированная точка на фазовом портрете уравнений РГ должна располагаться рядом с гейзенберговской фиксированной точкой. В результате критические индексы, отвечающие этим точкам, должны почти совпадать друг с другом. Представление о степени их близости можно получить, сопоставляя, например, наиболее надежные численные значения индекса восприимчивости $\gamma$ для гейзенберговской и кубической точек; они равны соответственно 1.3895(50) [23] и 1.390(6) [14]. Совершенно очевидно, что, измеряя критические индексы в эксперименте, невозможно установить, какой из видов критического поведения реализуется в кубических ферромагнетиках.

Тем не менее выяснить с помощью физических или компьютерных экспериментов, как ведет себя система с кубической симметрией вблизи точки Кюри, все же можно, но для этого следует изучить нелинейные восприимчивости различных порядков $\chi^{(2 k)}[24]$. Как показал РГ-анализ, анизотропия нелинейной восприимчивости $\chi^{(4)}$ в критической области может достигать $5 \%$, что определенно позволяет считать ее измеримой [24]. В то же время к этому результату вряд ли можно относиться как ко вполне надежному, поскольку он получен путем обработки рядов для $\beta$-функций трехмерной кубической модели, коэффициенты которых не малы, а сами разложения, как известно, являются расходящимися.

В такой ситуации естественно обратиться к альтернативным теоретическим схемам, которые позволили бы найти анизотропию восприимчивости $\chi^{(4)}$ при $T \rightarrow T_{\mathrm{c}}$ и подтвердить или скорректировать оценку, приведенную выше. Наиболее попу- 
лярной среди них следует считать $\epsilon$-разложение, которое широко используется для вычисления критических индексов и других универсальных параметров критического поведения. Вторым теоретико-полевым методом, перспективным в указанном плане, является техника псевдо- $\epsilon$-разложения, предложенная много лет назад Б. Никелом (см. ссылку [19] в статье [25]). Эта техника показала свою исключительную эффективность при вычислении универсальных характеристик не только трехмерных [20], [23], [25]-[30], но и двумерных систем [25], [31]-[35], для которых известные РГ-разложения короче и более сильно расходятся. Применение метода псевдо- $\epsilon$-разложения настолько ускоряет сходимость итераций и сглаживает осцилляции численных оценок, что во многих случаях для получения надежных количественных результатов оказывается достаточным использование простых аппроксимант Паде или даже прямое суммирование соответствующих рядов.

Ниже мы найдем величину анизотропии кубических ферромагнетиков вблизи точки Кюри с помощью методов $\epsilon$-разложения и псевдо- $\epsilon$-разложения, сопоставим числа, которые получаются в результате применения трех вариантов техники теоретико-полевой РГ, и обсудим полученные результаты.

В критической области разложение свободной энергии кубической модели по степеням проекций намагниченности $M_{\alpha}$ может быть записано в виде

$$
F\left(M_{\alpha}, m\right)=F(0, m)+\frac{1}{2} m^{2-\eta} M_{\alpha}^{2}+m^{1-2 \eta}\left(u_{4}+v_{4} \delta_{\alpha \beta}\right) M_{\alpha}^{2} M_{\beta}^{2}+\cdots,
$$

где $m$ - обратная корреляционная длина, $\eta$ - индекс Фишера, а $u_{4}$ и $v_{4}$ - безразмер-

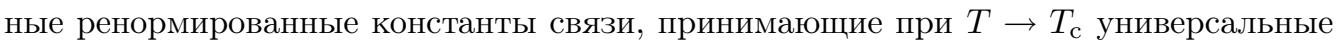
значения. Через эти константы, в частности, можно выразить и интересующую нас нелинейную восприимчивость четвертого порядка:

$$
\chi_{\alpha \beta \gamma \delta}^{(4)}=\left.\frac{\partial^{3} M_{\alpha}}{\partial H_{\beta} \partial H_{\gamma} \partial H_{\delta}}\right|_{H=0} .
$$

Особый интерес представляют значения нелинейной восприимчивости для двух высокосимметричных направлений, отвечающих ориентации внешнего поля вдоль ребpa $\left(\chi_{\mathrm{c}}^{(4)}\right)$ и пространственной диагонали $\left(\chi_{\mathrm{d}}^{(4)}\right)$ элементарной ячейки. Для этих направлений разность значений $\chi^{(4)}$ максимальна, т. е. кубическая анизотропия выражена наиболее сильно. Как легко показать,

$$
\chi_{\mathrm{c}}^{(4)}=-24 \frac{\chi^{2}}{m^{3}}\left(u_{4}+v_{4}\right), \quad \chi_{\mathrm{d}}^{(4)}=-24 \frac{\chi^{2}}{m^{3}}\left(u_{4}+\frac{v_{4}}{3}\right),
$$

где $\chi$ - линейная восприимчивость. Естественной характеристикой анизотропии нелинейной восприимчивости является отношение

$$
\delta^{(4)}=\frac{\left|\chi_{\mathrm{c}}^{(4)}-\chi_{\mathrm{d}}^{(4)}\right|}{\chi_{\mathrm{c}}^{(4)}} .
$$

Это отношение можно записать как

$$
\delta^{(4)}=\frac{2 v_{4}}{3\left(u_{4}+v_{4}\right)}
$$


и выразить через параметр анизотропии

$$
A=\frac{v_{4}}{u_{4}}
$$

Его величину $A^{*}$ в точке Кюри мы и будем искать в настоящей работе.

Флуктуационный гамильтониан кубической модели имеет вид

$$
H=\frac{1}{2} \int d^{D} x\left[m_{0}^{2} \varphi_{\alpha}^{2}+\left(\nabla \varphi_{\alpha}\right)^{2}+\frac{u_{0}}{12} \varphi_{\alpha}^{2} \varphi_{\beta}^{2}+\frac{v_{0}}{12} \varphi_{\alpha}^{4}\right],
$$

где $\varphi_{\alpha}-n$-компонентное поле флуктуаций параметра порядка, квадрат голой массы $m_{0}^{2} \sim T-T_{\mathrm{c}}^{(0)}$, а $T_{\mathrm{c}}^{(0)}$ - температура фазового перехода в приближении среднего поля. Асимптотическое значение параметра анизотропии в критической точке $A^{*}$ определяется координатами $u_{4}^{*}$ и $v_{4}^{*}$ кубической фиксированной точки уравнений РГ. Для $D=4-\epsilon$ они известны сегодня в пятипетлевом приближении [15]. Соответствующие $\epsilon$-разложения для физически интересного случая $n=3$ выглядят следующим образом:

$$
\begin{aligned}
& u^{*}=1.22222222 \epsilon+0.49291267 \epsilon^{2}-0.4899848 \epsilon^{3}+0.5912287 \epsilon^{4}-1.429021 \epsilon^{5}, \\
& v^{*}=-0.40740741 \epsilon+0.19783570 \epsilon^{2}+0.2270881 \epsilon^{3}+0.1855026 \epsilon^{4}-0.6908416 \epsilon^{5},
\end{aligned}
$$

где $u^{*}=(11 / 2 \pi) u_{4}^{*}, v^{*}=(11 / 2 \pi) v_{4}^{*}$. Видно, что разложения $(8),(9)$ имеют нерегулярную структуру (не являются знакопеременными), их коэффициенты не малы и быстро начинают расти с ростом номера члена ряда. Неудивительно поэтому, что использование различных методов пересуммирования, в том числе основанных на преобразовании Бореля, не ведет в данном случае к сколь-нибудь удовлетворительным результатам. Отталкиваясь от рядов для констант связи, можно, однако, получить $\epsilon$-разложение для самого́ параметра анизотропии,

$$
A^{*}=-\frac{1}{3}+0.29629630 \epsilon-0.0673269 \epsilon^{2}+0.458956 \epsilon^{3}-1.31038 \epsilon^{4},
$$

которое, будучи тоже расходящимся, имеет перед (8), (9) то преимущество, что является знакопеременным. Поскольку старшие коэффициенты в разложении (10) сильно растут, для обработки этого ряда естественно применить преобразование Бореля с последующим аналитическим продолжением борелевского образа с помощью аппроксимант Паде [L/M].

Результаты пересуммирования ряда (10) с помощью этой техники приведены в табл. 1. Хотя полученные оценки довольно сильно разбросаны, наиболее вероятное значение $A^{*}$, т. е. то, которое дается диагональной аппроксимантой $[2 / 2]$, лежит недалеко от интервала, в котором группируются результаты обработки шестипетлевых РГ-разложений.

Действительно, пересуммирование рядов для $\beta$-функций трехмерной кубической модели ведет к значениям $u_{4}^{*}=0.755 \pm 0.010, v_{4}^{*}=0.067 \pm 0.014$ [14], откуда, в свою очередь, следует, что $A^{*}=0.089 \pm 0.018$. С другой стороны, различие полученных чисел (0.089 и 0.124) не столь мало, чтобы полностью его игнорировать. У этого расхождения должны быть причины. Одной из них можно считать специфическую особенность $\epsilon$-разложения для $A^{*}$ : при физическом значении $\epsilon$ первые два члена ряда (10) почти компенсируют друг друга, что эффективно укорачивает этот 
ТАБлицА 1. Треугольник Паде-Бореля для $\epsilon$-разложения (10) универсального значения параметра анизотропии $A^{*}$. Аппроксиманта $[1 / 2]$ имеет "опасный” полюс, поэтому соответствующее число в таблице отсутствует.

\begin{tabular}{l|c|ccccc} 
& \multicolumn{1}{c|}{ L } & 0 & 1 & 2 & 3 & 4 \\
\hline 0 & -0.3333 & -0.0370 & -0.1044 & 0.3546 & -0.9558 \\
1 & -0.2063 & -0.0882 & -0.0482 & 0.0314 & \\
2 & -0.1737 & - & 0.1241 & & \\
3 & -0.1568 & -0.1110 & & & \\
4 & -0.1489 & & & &
\end{tabular}

ряд и резко ухудшает его аппроксимирующие свойства. Поскольку $\epsilon$-разложения для констант связи также имеют неблагоприятную в вычислительном отношении структуру, мы приходим к выводу, что метод $\epsilon$-разложения не позволяет оценить с достаточной точностью величину анизотропии кубического ферромагнетика в критической области.

В такой ситуации разумно обратиться к другому варианту РГ-теории возмущений - к технике псевдо- $\epsilon$-разложения. Основная идея этого метода состоит в том что, отталкиваясь от трехмерных РГ-разложений, мы вводим в теорию формальный малый параметр $\tau$, поставив его перед линейными членами в рядах для $\beta$-функций, и ищем наблюдаемые в виде разложений по $\tau$. Их численные значения можно затем получить, положив $\tau=1$. Итак, будем исходить из шестипетлевых РГ-разложений для кубической модели [14]. Применяя описанную выше итерационную процедуру к системе уравнений

$$
\beta_{u}(u, v)=0, \quad \beta_{v}(u, v)=0,
$$

для координат кубической фиксированной точки при $n=3$ находим

$$
\begin{aligned}
u^{*}=1.2222222 \tau+ & 0.3323342 \tau^{2}-0.122585 \tau^{3}- \\
- & 0.065595 \tau^{4}-0.061083 \tau^{5}+0.01269 \tau^{6}, \\
v^{*}=-0.4074074 \tau & +0.1306486 \tau^{2}+0.232337 \tau^{3}+ \\
& +0.128399 \tau^{4}+0.050252 \tau^{5}+0.02224 \tau^{6} .
\end{aligned}
$$

Эти ряды имеют структуру значительно более привлекательную, чем $\epsilon$-разложения (8), (9). Их старшие коэффициенты малы и убывают по модулю с ростом номера члена ряда. Можно ожидать, что уже прямое суммирование рядов (12), (13) даст приемлемые результаты.

Действительно, подставляя в разложение (12) $\tau=1$, мы получим $u^{*}=1.318$, число, близкое к наиболее надежной - шестипетлевой - РГ-оценке $u^{*}=1.321$ [14]. Аналогичная процедура для второй константы связи дает $v^{*}=0.1565$. Это значение уже существенно отличается от своего шестипетлевого РГ-аналога $v^{*}=0.117$ [14], давая, однако, для параметра анизотропии величину 0.119 , мало отличающуюся от той, которую мы извлекли из $\epsilon$-разложения для $A^{*}$. 
ТАБлицА 2. Треугольник Паде для псевдо- $\epsilon$-разложения (12) константы связи $u^{*}$. Аппроксиманты [3/1], [3/2] и [2/2] имеют полюсы, близкие к единице, и поэтому соответствующее оценки ненадежны; в таблице они взяты в скобки. Нижняя строка RoC показывает темп и характер сходимости оценок Паде к асимптотическому значению. Здесь оценка Паде $k$-го порядка есть число, которое дает соответствующая диагональная аппроксиманта $[\mathrm{L} / \mathrm{L}]$, либо полусумма величин, даваемых аппроксимантами $[\mathrm{L} / \mathrm{L}-1]$ и $[\mathrm{L}-1 / \mathrm{L}]$, когда диагональная аппроксиманта отсутствует. Результирующее значение $u^{*}=1.3218$ получено усреднением по трем рабочим аппроксимантам [4/1], [2/3] и [1/4].

\begin{tabular}{|c|c|c|c|c|c|c|}
\hline $\mathrm{M}$ & 0 & 1 & 2 & 3 & 4 & 5 \\
\hline 0 & 1.2222 & 1.5546 & 1.4320 & 1.3664 & 1.3053 & 1.3180 \\
\hline 1 & 1.6787 & 1.4650 & 1.2909 & $(0.4782)$ & 1.3158 & \\
\hline 2 & 1.3545 & 1.3832 & $(0.9025)$ & $(1.2483)$ & & \\
\hline 3 & 1.3868 & 1.3629 & 1.3197 & & & \\
\hline 4 & 1.3004 & 1.3300 & & & & \\
\hline 5 & 1.3472 & & & & & \\
\hline $\mathrm{RoC}$ & 1.2222 & 1.6166 & 1.4650 & 1.3371 & $(0.9025)$ & 1.3218 \\
\hline
\end{tabular}

ТАБлицА 3. Треугольник Паде для псевдо-є-разложения (13) константы связи $v^{*}$. Аппроксиманта $[1 / 1]$ имеет полюс, близкий к единице, соответствующая оценка ненадежна, и в таблице она взята в скобки. Нижняя строка RoC и оценка Паде $k$-го порядка рассчитываются так же, как и в случае константы $u^{*}$ (см. табл. 2).

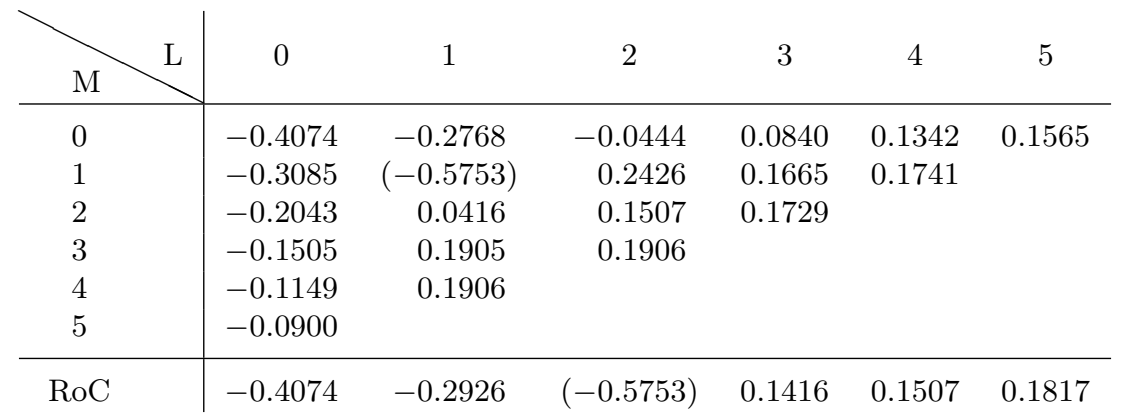

Попробуем уточнить оценки, получаемые в рамках метода псевдо- $\epsilon-$ разложения, с помощью аппроксимант Паде. Треугольники Паде для разложений (12), (13) приведены в табл. 2 и 3. Видно, что, хотя три старшие аппроксиманты для $u^{*}$, включая $[3 / 2]$, имеют опасные полюсы, итерационная процедура в целом устойчиво сходится к значению 1.322, очень близкому к шестипетлевой РГ-оценке $u^{*}=1.321$ [14]. Это значение мы и примем в качестве окончательного. Структура содержимого табл. 3 также вполне регулярна в том смысле, что старшие аппроксиманты Паде дают оценки для $v^{*}$, отличающиеся от асимптотического значения $v^{*}=0.1817$ не более чем на $0.03 \div 0.04$. Взяв найденные значения координат кубической фиксированной точки, получим $A^{*}=0.1375$. Это число представляет несомненный инте- 
ТАБлицА 4. Треугольник Паде для псевдо-є-разложения (14) параметра анизотропии в точке Кюри. Аппроксиманты [2/1], [1/2], [4/1] и [1/4] имеют опасные полюсы, соответствующие оценки ненадежны и поэтому взяты в скобки. Нижняя строка $\mathrm{RoC}$ и оценка Паде $k$-го порядка рассчитываются так же, как и в табл. 2 и 3.

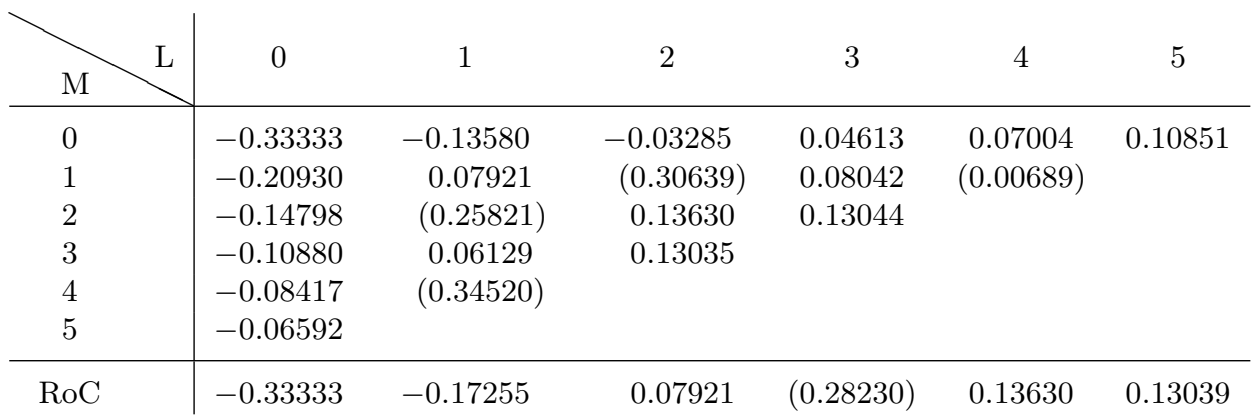

pec: оно сильно отличается от шестипетлевой РГ-оценки $A^{*}=0.117 / 1.321=0.0886$ и ощутимо превышает значение $A^{*}=0.119$, полученное прямым суммированием псевдо- $\epsilon$-разложений для $u^{*}$ и $v^{*}$.

Найдем далее альтернативную оценку параметра анизотропии в критической точке. Это можно сделать, построив псевдо- $\epsilon$-разложение непосредственно для $A^{*}$. Комбинируя ряды (12) и (13), получим

$$
A^{*}=-\frac{1}{3}+0.197531 \tau+0.102951 \tau^{2}+0.078982 \tau^{3}+0.023907 \tau^{4}+0.03847 \tau^{5} .
$$

Коэффициенты этого ряда, за исключением последнего, убывают по модулю, последний коэффициент мал, и, следовательно, разложение (14) пригодно для получения численных оценок. Результаты его суммирования с помощью аппроксимант Паде приведены в табл. 4. Как видно, две из старших аппроксимант, [4/1] и [1/4], имеют опасные полюсы, а асимптотическое значение $A^{*}=0.1304$ близко к ранее полученному числу 0.1375 . Взяв среднее этих величин и считая их разность естественной оценкой точности используемой аппроксимационной схемы, можно положить $A^{*}=0.134 \pm 0.004$. С другой стороны, псевдо- $\epsilon$-разложения для $v^{*}$ и $A^{*}$ имеют неприятную особенность: их первые члены отрицательны и максимальны по модулю, вследствие чего численные результаты получаются как малые разности больших чисел. Это несомненно снижает их точность. Поэтому мы примем в качестве окончательной более консервативную оценку

$$
A^{*}=0.13 \pm 0.01
$$

которая представляется нам реалистичной.

Итак, метод псевдо- $\epsilon$-разложения дает значение параметра анизотропии, которое в полтора раза отличается от своего РГ-аналога $A^{*}=0.089$. Чем можно объяснить столь существенное расхождение двух теоретико-полевых оценок, полученных в максимально высоком на сегодня - шестипетлевом - приближении? Одна из возможных причин уже упоминалась: численное значение $A^{*}$ много меньше коэффициентов при первых членах используемых рядов, и оно ищется как малая разность 
больших чисел. Эта малость, в свою очередь, связана с тем, что граничная размерность параметра порядка $n_{\text {c }}$ близка к 3 . Если бы $n_{\mathrm{c}}$ совпадала с физическим значением $n$, то параметр анизотропии в критической точке был бы равен нулю.

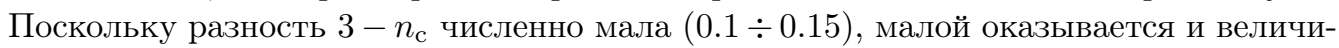
на $A^{*}$. Но именно для этой разности разные теоретико-полевые схемы дают ощутимо различающиеся оценки. Так, обработка с помощью техники конформ-борель шестипетлевых трехмерных РГ-разложений для $\beta$-функций кубической модели ведет к значению $3-n_{\mathrm{c}}=0.11$ [14], в то время как значения этой разности, полученные пересуммированием псевдо- $\epsilon-$ и $\epsilon$ - разложений для $n_{\mathrm{c}}$, составляют соответственно 0.138 [20] и 0.145 [18]. Поскольку первое из приведенных чисел отличается от двух других на десятки процентов, неудивительно, что различие значений $A^{*}$, полученных в рамках тех же итерационных схем, оказывается столь значительным.

В заключение отметим, что если принять найденное выше значение $A^{*}$, то анизотропия нелинейной восприимчивости

$$
\delta^{(4)}=\frac{2 A}{3(1+A)}
$$

в точке Кюри возрастет почти до 8\%. Это делает аргументацию в пользу возможности экспериментального обнаружения анизотропного критического поведения кубических ферромагнетиков [24] еще более убедительной.

\section{Список литературы}

[1] K. G. Wilson, M. E. Fisher, "Critical exponents in 3.99 dimensions", Phys. Rev. Lett., 28:4 (1972), 240-243.

[2] D. J. Wallace, "Critical behaviour of anisotropic cubic systems", J. Phys. C, 6:8 (1973), 1390-1404.

[3] I. J. Ketley, D. J. Wallace, "A modified epsilon expansion for a Hamiltonian with cubic point-group symmetry", J. Phys. A: Math. Gen., 6:11 (1973), 1667-1678.

[4] И.Ф. Люксютов, В.Л. Покровский, "Фазовые переходы первого рода в системах с кубической анизотропией”, Писъма в ЖЭТФ, 21:1 (1975), 22-25.

[5] A. Aharony, "Critical behavior of anisotropic cubic systems", Phys. Rev. B, 8:9 (1973), 4270-4273.

[6] А.И. Соколов, "О фазовом переходе в трехмерной модели. Влияние кубической анизотропии", ФТT, 19:3 (1977), 747-755.

[7] K. E. Newman, E. K. Riedel, "Cubic $N$-vector model and randomly dilute Ising model in general dimensions", Phys. Rev. B, 25:1 (1982), 264-280; Erratum, 30:5, 2924-2924.

[8] M. Ferer, J. P. Van Dyke, W. J. Camp, "Effect of a cubic crystal field on the critical behavior of a 3D model with Heisenberg exchange coupling: a high-temperature series investigation", Phys. Rev. B, 23:5 (1981), 2367-2373.

[9] M. Tissier, D. Mouhanna, J. Vidal, B. Delamotte, "Randomly dilute Ising model: A nonperturbative approach", Phys. Rev. B, 65:14 (2002), 140402, 4 pp., arXiv: cond-mat/0109176.

[10] И. О. Майер, А.И. Соколов, "О критическом поведении кубических кристаллов при структурных фазовых переходах", Изв. АН СССР. Сер. физ., 51:12 (1987), 2103-2106; I. O. Maier, A. I. Sokolov, "Is a cubic crystal "isotropic" in the critical point?", Ferroelectrics Lett. Sect., 9:4 (1988), 95-98.

[11] N. A. Shpot, "Critical behavior of the $m n$-component field model in three dimensions II. Three-loop results", Phys. Lett. A, 142:8-9 (1989), 474-478. 
[12] I. O. Mayer, A. I. Sokolov, B. N. Shalaev, "Critical exponents for cubic and impure uniaxial crystals: most accurate (?) theoretical values", Ferroelectrics, 95 (1989), 93-96.

[13] D. V. Pakhnin, A.I. Sokolov, "Five-loop renormalization-group expansions for the three-dimensional $n$-vector cubic model and critical exponents for impure Ising systems", Phys. Rev. B, 61:22 (2000), 15130-15135, arXiv: cond-mat/9912071.

[14] J. M. Carmona, A. Pelissetto, E. Vicari, " $N$-component Ginzburg-Landau Hamiltonian with cubic anisotropy: a six-loop study", Phys. Rev. B, 61:22 (2000), 15136-15151, arXiv: cond-mat/9912115.

[15] H. Kleinert, V. Schulte-Frohlinde, "Exact five-loop renormalization group functions of $\phi^{4}$-theory with $O(N)$-symmetric and cubic interactions. Critical exponents up to $\varepsilon^{5} "$, Phys. Lett. B, 342:1-4 (1995), 284-296, arXiv: cond-mat/9503038.

[16] H. Kleinert, S. Thoms, "Large-order behavior of a two-coupling-constant $\varphi^{4}$ theory with cubic anisotropy", Phys. Rev. D, 52:10 (1995), 5926-5943, arXiv: hep-th/9508172.

[17] H. Kleinert, S. Thoms, V. Schulte-Frohlinde, "Stability of a three-dimensional cubic fixed point in the two-coupling-constant $\varphi^{4}$ theory", Phys. Rev. B, 56:22 (1997), 14428-14434, arXiv: quant-ph/9611050.

[18] B. N. Shalaev, S. A. Antonenko, A. I. Sokolov, "Five-loop $\sqrt{\varepsilon}$-expansion for random Ising model and marginal spin dimensionality for cubic systems", Phys. Lett. A, 230:1-2 (1997), 105-110, arXiv: cond-mat/9803388.

[19] R. Folk, Yu. Holovatch, T. Yavors'kii, "Effective and asymptotic critical exponents of a weakly diluted quenched Ising model: Three-dimensional approach versus $\sqrt{\varepsilon}$ expansion", Phys. Rev. B, 61:22 (2000), 15114-15129, arXiv: cond-mat/9909121.

[20] R. Folk, Yu. Holovatch, T. Yavors'kii, "Pseudo- $\varepsilon$ expansion of six-loop renormalization-group functions of an anisotropic cubic model", Phys. Rev. B, 62:18 (2000), 12195-12200, arXiv: cond-mat/0003216; Erratum, 63:18, 189901.

[21] K. B. Varnashev, "Stability of a cubic fixed point in three dimensions: Critical exponents for generic N", Phys. Rev. B, 61:21 (2000), 14660-14674, arXiv: cond-mat/9909087.

[22] A. Pelissetto, E. Vicari, "Critical phenomena and renormalization-group theory", Phys. Rep., 368:6 (2002), 549-727, arXiv: cond-mat/0012164.

[23] R. Guida, J. Zinn-Justin, "Critical exponents of the N-vector model", J. Phys. A: Math. Gen., 31:40 (1998), 8103-8121, arXiv: cond-mat/9803240.

[24] D. V. Pakhnin, A.I. Sokolov, "Renormalization group and nonlinear susceptibilities of cubic ferromagnets at criticality", Phys. Rev. B, 64:9 (2001), 094407, 6 pp., arXiv: cond-mat/0102368.

[25] J. C. Le Guillou, J. Zinn-Justin, "Critical exponents from field theory", Phys. Rev. B, 21:9 (1980), 3976-3998.

[26] Yu. Holovatch, D. Ivaneiko, B. Delamotte, "On the criticality of frustrated spin systems with noncollinear order", J. Phys. A: Math. Gen., 37:11 (2004), 3569-3575, arXiv: cond-mat/0312260.

[27] A. I. Sokolov, M. A. Nikitina, "Pseudo- $\varepsilon$ expansion and renormalized coupling constants at criticality", Phys. Rev. E, 89:5 (2014), 052127, 10 pp., arXiv: 1402.3531.

[28] A.I. Sokolov, M. A. Nikitina, "Fisher exponent from pseudo- $\varepsilon$ expansion", Phys. Rev. E, 90:1 (2014), 012102, 5 pp., arXiv: 1402.3894.

[29] М. А. Никитина, А. И. Соколов, "Критические индексы и псевдо-є-разложение", ТМФ, 186:2 (2016), 230-242, arXiv: 1602.08681.

[30] A.I. Sokolov, M.A. Nikitina, "Pseudo- $\epsilon$ expansion and critical exponents of superfluid helium", Phys. A, 444 (2016), 177-181, arXiv: 1402.4318.

[31] P. Calabrese, E. V. Orlov, D.V. Pakhnin, A.I. Sokolov, "Critical behavior of two-dimensional cubic and $M N$ models in the five-loop renormalization group approximation", Phys. Rev. B, 70:9 (2004), 094425, 15 pp., arXiv: cond-mat/0405432. 
[32] P. Calabrese, P. Parruccini, "Harmonic crossover exponents in $O(n)$ models with the pseudo- $\varepsilon$ expansion approach", Phys. Rev. B, 71:6 (2005), 064416, 8 pp., arXiv: cond-mat/0411027.

[33] А. И. Соколов, "Псевдо-є-разложение и двумерная модель Изинга", $\Phi T T, 47: 11$ (2005), 2056-2059, arXiv: cond-mat/0510088.

[34] А.И. Соколов, “Фазовые переходы в двумерных системах и многопетлевые ренормгрупповые разложения", ТМФ, 176:1 (2013), 140-149.

[35] M. A. Nikitina, A. I. Sokolov, "Critical exponents in two dimensions and pseudo- $\varepsilon$ expansion", Phys. Rev. E, 89:4 (2014), 042146, 6 pp., arXiv: 1312.1062. 